\title{
Effects of respiratory muscle training and electrical stimulation of abdominal muscles on respiratory capabilities in tetraplegic patients
}

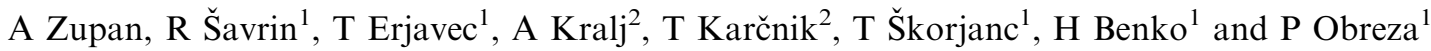 \\ ${ }^{1}$ Rehabilitation Institute, 1000 Ljubljana, Linhartova 51, Slovenia; ${ }^{2}$ University of Ljubljana - Faculty of electrical and \\ computer engineering, 1000 Ljubljana, Tržaška 25, Slovenia
}

\begin{abstract}
Thirteen tetraplegic patients were included in the study of the effects of respiratory muscle training and of electrical stimulation of the abdominal muscles on their respiratory capabilities. Each patient was subjected for three 1 month lasting periods of the study: for inspiratory muscle training, expiratory muscle training and for a period without training. The sequence of these three periods was random for each patient. Respiratory tests (RT) measuring forced vital capacity $(\mathrm{FVC})$ and forced expiratory volume in one second $\left(\mathrm{FEV}_{1}\right)$ were conducted before and following each monthly period. Measurements were taken under four sets of conditions: the patients' unassisted efforts, their efforts combined with pressure manually applied by a therapist to the upper part of their abdomen, and their efforts accompanied by electrical stimulation (ES) of the abdominal muscles during the early phase of expirium, once triggered by the therapist and once by the patients themselves. RT values were increased following respiratory muscle training and inspiratory training apparently had a slightly greater effect than its expiratory counterpart. The increments of values of RT were statistically significant $(P<0.05)$ after the inspiratory muscle training. RT measurements were greater when the patient's voluntary effort was combined with ES of abdominal muscles than when it was not. This study concludes that respiratory muscle training is a potentially effective approach and that ES of the abdominal muscles has potentials to improve coughing in tetraplegic patients.
\end{abstract}

Keywords: tetraplegic patients; respiratory capabilities; respiratory muscle training; cough; electrical stimulation

\section{Introduction}

Tetraplegia resulting from acute cervical spinal cord trauma is often associated with profound respiratory compromise. Injuries at or above the cord segments $\mathrm{C} 3$ to $\mathrm{C} 5$ involve the phrenic nerves and cause partial or complete bilateral hemidiaphragmatic paralysis. In addition, intercostal muscle paralysis caudal to the lesion limits the normal outward expansion of the middle and upper rib cage, further compromising inspiration. Expiration is also greatly reduced by paralysis of the abdominal and other expiratory muscles. Sternocleidomastoid, scalene and trapezoid activity persists in high cord injuries; however, their efficiency is greatly reduced. ${ }^{1}$ Because of their extensive respiratory muscle dysfunction, those with high cervical tetraplegia are unable to generate an adequate vital capacity due to reduced inspiratory and expiratory functions. Hypoxemia is common and results from both hypoventilation and microatelectasis. ${ }^{2}$

Tetraplegic patients with lesions in the lower cervical cord, whose phrenic nerve nuclei are com-

Correspondence: A Zupan pletely or partially intact, can contract their diaphragms to variable extent. Nevertheless, they lack the intercostal muscle activity necessary to stabilise the rib cage so that the hemidiaphragms can function properly; as a result, their inspiratory function is compromised. Like higher level tetraplegic patients they have also lost the use of their abdominal and other expiratory muscles. This combination of expiratory and inspiratory weakness prevents them from coughing and clearing secretions, placing them at high risk for respiratory tract infections. ${ }^{1}$

Our study sought to evaluate whether the prescribed and here described respiratory muscle training could improve the respiratory capacities in tetraplegic patients and whether electrical stimulation (ES) of abdominal muscles in the early phase of expirium could increase the efficacy of coughing.

\section{Materials and methods}

The study included 13 tetraplegic patients, 11 men and two women, aged from 17 to 46 years, averaging 26.9 years. The neurological motor level of spinal cord 
lesion was at $\mathrm{C} 4$ in two patients, $\mathrm{C} 5$ in two patients, C6 in six patients and at $\mathrm{C} 7$ in three patients. Ten patients had complete and three incomplete lesion of the cervical spinal cord. The cause of lesion was traffic injury in nine patients, diving injury in three patients and cavernoma medullae spinalis in one patient.

Prior to starting the study, the patients were fully acquainted with the purpose and course of the study and they confirmed their voluntary participation. All patients were familiar with spirographic tests to which they had been subjected in the past. The breathing exercises were demonstrated and the measuring procedures were explained. None of the patients had histories of lung disease before the trauma. Lung roentgenograms and ECG were found normal at the entry into the study and chest physical examination revealed no signs of respiratory disease at the time of the study. The time between injury and the beginning of the study for 12 patients with traumatic injury was from 3 to 12 months, 7 months on average. The patient with cavernoma had a history of disease for 10 years. Patients with a spinal cord injury are usually accepted into our institute from traumatology clinics 2-3 months after injury. While at such clinics they undergo a program of proper nursing, passive muscle exercises and respiratory therapy designed to prevent secondary pulmonary complications. After acceptance into our institute these patients begin an intensive physiotherapy program (mainly assisted exercises). This includes some respiratory muscle exercises, but much less than that to which the patients in our study were subjected.

Each patient was subjected for three 1 month lasting periods during the study: inspiratory muscle training, expiratory muscle training and a period without training. The sequence of these three periods was random for each patient. We considered 1 month of training as sufficient time to show some results. Both programs of respiratory muscle training consisted of seven different exercises and each exercise was repeated ten times. Each training session lasted $20-$ $30 \mathrm{~min}$ and was conducted twice a day six days a week for a period of 4 weeks. Inspiratory muscle training consisted of inspiratory muscles exercises for training both strength and endurance. The exercises were: the patient inhales slowly, holds his breath and exhales slowly, each phase lasting approximately $5 \mathrm{~s}$; the patient tries to reach maximal value on the incentive spirometer with the breath as deep as possible no matter how quick this procedure is done; the patient takes an average value of eight maximal inspirations and tries to hold the breath at $75 \%$ of this value as long as he can; etc. Expiratory muscle training consisted of expiratory muscle exercises accompanied by ES of the abdominal muscles. The exercises were: the patient inhales slowly, holds his breath and exhales slowly, the same as in inspiratory muscle training; tries to reach maximal expiration on Wrights peak flow meter, two times without ES and eight times with ES; blows air bubbles through the water with a thin straw for $3 \mathrm{~min}$; etc. During expiratory muscle training neither P-Flex nor weights were used for strengthening abdominal muscles. About $30 \mathrm{ES}$ were applied during each training session, lasting 20-30 min. A portable battery powered stimulator and two pairs of surface electrodes were employed. The stimulator delivered a single pulse train of $0.75 \mathrm{~s}$ duration. Individual pulses were $0.3 \mathrm{~ms}$ wide, delivered at a $50 \mathrm{~Hz}$ stimulation frequency. The amplitude was adjusted for a visible and medium strong contraction or up to the level which was tolerated by the patient not causing any irritation or discomfort up to $110 \mathrm{~V}$. The same stimulation was applied when training patients and measuring results. Besides the above described respiratory muscle training all patients in our study had a usual physiotherapy program during all 3 months of the study.

Respiratory tests (RT) - forced vital capacity (FVC) and forced expiratory volume in one second (FEV1) were conducted in sitting and lying positions previous to study and at the conclusion of each 1 month period. RT were conducted under four sets of conditions: the patient's unassisted effort, the patient's effort accompanied by a therapist's manual assistance, and the patient's effort accompanied by ES of the abdominal muscles; the stimulation was triggered by a command switch once by the therapist and once by the patient himself in the early phase of expirium. The manual assistance and the triggering of stimulation was always done by the same therapist. The best of three readings of each measurement was used.

At the beginning of the study each patient was given a $\log$ where he registered the times and durations of each day's respiratory training and ES, as well as any special effects, such as cough or sputum production, changes in skin under the electrodes and any subjective remarks regarding breathing etc. The patients were training under the supervision of the therapists, who also offered help to those patients who needed it. Neither a corset nor abdominal binder were used during the respiratory muscle training or during measurements of RT in the sitting position.

Statistical comparisons of the measured RT before the beginning of the study and after each 1 month period were carried out with Student's $t$ test, and 5\% was chosen as the level of significance.

\section{Results}

The results of RT measured before the beginning of the study and at the conclusion of each 1 month period under four different conditions of measurements can be seen in Figures 1 to 4 . The RT were measured in sitting and lying position. In the Figures 1 to 4 the average values of the best of three attempts for RT for all 13 patients are expressed as percentages of the initial values measured in the sitting position before the beginning of the study.

At the trials of the patient's unassisted effort the measured RT in both positions - sitting and lying 
revealed statistically significant increase $(P<0.05)$ after the inspiratory muscle training. The average increase of FVC in sitting position at the conclusion of inspiratory muscle training was $19 \%$ (the values were greater in 11 out of 13 patients, the greatest increase was $60 \%$ ); the average increase in lying position was $17.5 \%$ (greater values in 10 out of 13 patients). The average increase of FEV1 after the conclusion of inspiratory training was $20.5 \%$ in sitting and $16 \%$ in lying position. At the conclusion of expiratory muscle training the measured RT revealed no statistically significant changes in sitting position but statistically significant changes in lying position of both FVC and FEV1. FVC measured in lying position increased $17 \%$ and FEV $116 \%$ on average.

At the trials when the patient's voluntary effort was combined with the therapist's manual assistance and where it was combined with ES triggered by the patient himself, the measured RT in both positions, sitting and lying, revealed stastistically significant increase $(P<0.05)$ following both kinds of training. At the trials when the patient's voluntary effort was combined with ES triggered by the therapist, there was a significant increase $(P<0.05)$ in measured RT in sitting and lying position after the period of inspiratory muscle training but no significant increase after the expiratory muscle training. After the period without training no one measurement showed significant increase in RT.

The values of RT were greater measured in lying than in sitting position; at the trials of the patient's unassisted effort, $\mathrm{FVC}$ for $9.5 \%$ on average (greater in nine and smaller in four patients) and FEV1 for $7 \%$ on average (greater in eight and smaller in five patients).

The greatest values of RT before the beginning of the study and at the end of each 1 month period were measured at trials of patient's effort accompanied by
FVC max - sitting

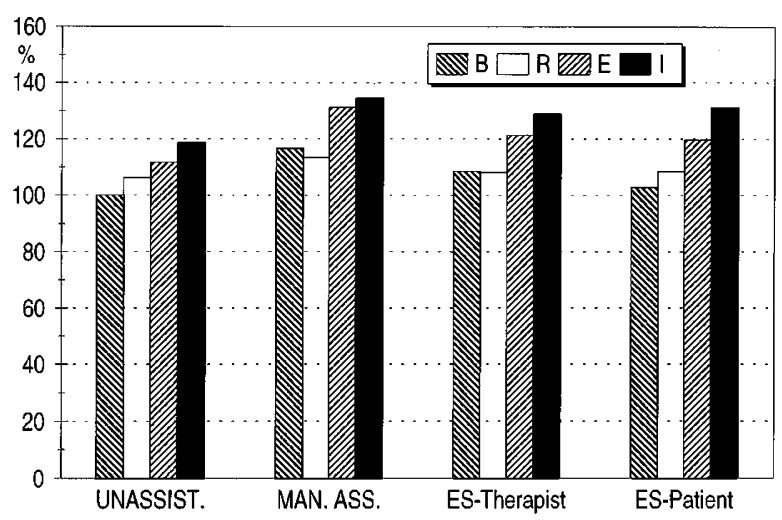

FVC max - lying

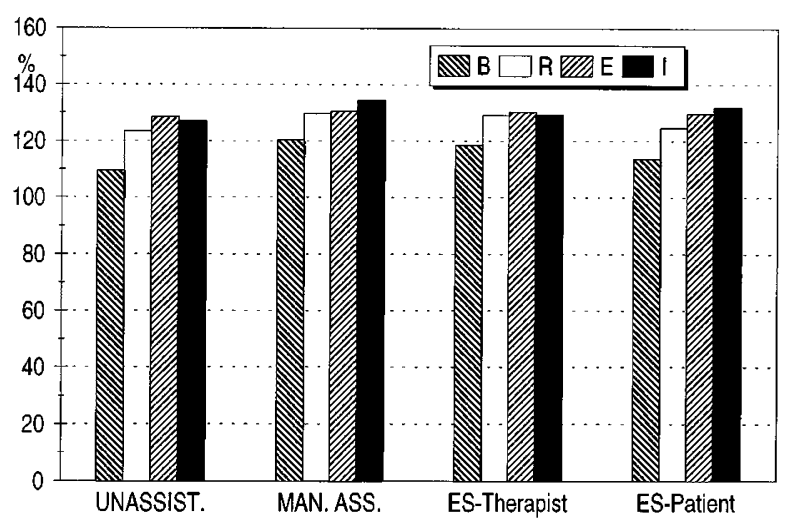

FEV1 max - sitting

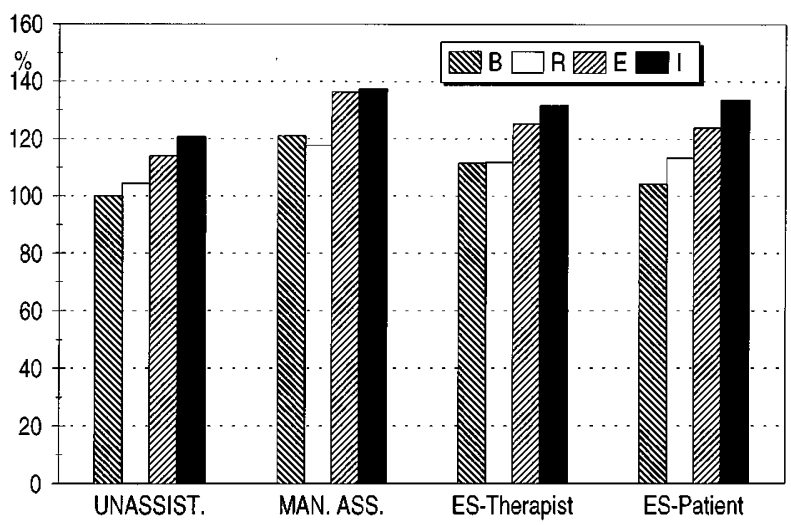

FEV1 max - lying

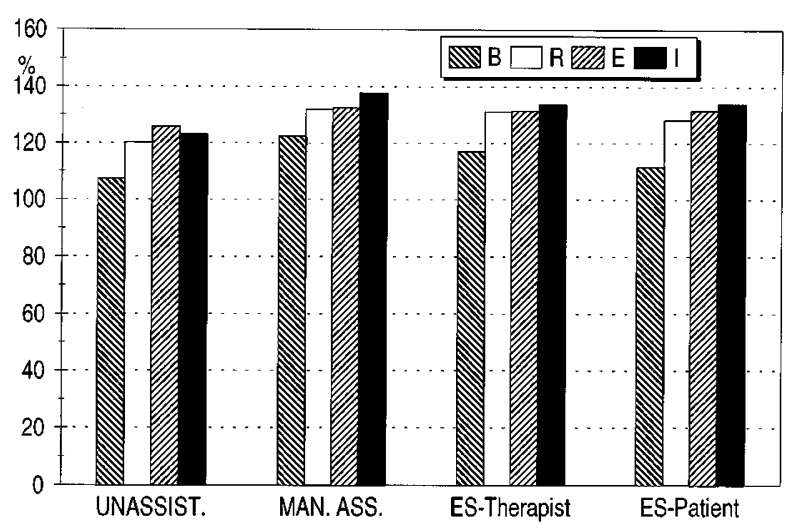

Figures 1-4 Measurements of forced vital capacity (FVC) and forced expiratory volume in one second (FEV1) in sitting and lying positions, before initiating muscle training (B), at the conclusion of 1 month period without training (R), at the conclusion of 1 month of expiratory muscle training (E) and at the conclusion of inspiratory muscle training (I). The following were measured: unassisted voluntary effort (UNASSIST), patient's effort accompanied by therapist's manual assistance (MAN ASS) and patient's effort accompanied by electrical stimulation of the abdominal muscles - triggered by the therapist (ES-Therapist), and triggered by the patient himself (ES-Patient). In the Figures 1 to 4 the average values of the best of three attempts for RT for all 13 patients are expressed as percentages of the initial values as measured in the sitting position before the beginning of the study 
the therapist's manual assistance. The patient's voluntary effort combined with ES was more effective than voluntary effort alone; this is true for both measurement conditions, when the stimulation was triggered by the therapist and when it was triggered by the patient himself.

The patients have performed their trainings very regularly as expected since all trainings were supervised by therapists. Two patients complained of experiencing more spasms during the expiratory muscle training combined with ES. More patients developed spasticity with increased reflex activity in the extremities during the measurements of RT especially at the measurements where patients voluntary effort was combined with the therapist's manual assistance or ES. Two patients noticed increased sputum production during both periods of training. They were both smokers. Three patients found the training sessions exhausting. Seven patients found that breathing was easier during the training periods; five patients asked to proceed with respiratory training after the conclusion of the study.

\section{Discussion}

The respiratory capabilities of 13 tetraplegic patients were investigated in this study with the aim to determine whether respiratory muscle training can improve these capabilities and whether cough efficiency can be improved through ES of abdominal muscles during the early phase of expirium. The patients' respiratory capabilities were assessed by RT where FVC and FEV1 were measured. FVC was chosen because it is one of the most frequently used pulmonary functional tests. It depends directly upon the strength of the respiratory muscles and pulmonary compliance. ${ }^{3}$ FVC is also a good estimation of cough efficiency since the mean peak flow during the cough is comparable to the mean peak flow during a FVC test. ${ }^{4}$ The FEV1 values were used to assess the extent to which the functioning of the expiratory muscles was preserved and indirectly for the assessment of the capacity to expectorate since FEV1 is known to depend primarily on the strength of the expiratory muscles and the diameter of the upper airways.

RT values were greater for almost all patients following respiratory muscle training. And this might be attributed to the training's influence upon muscle weakness or its influence on the mechanical properties of the lung and chest wall. Increased muscle strength and endurance could result in greater RT values. It is also possible that respiratory muscle training could affect the chest wall compliance which is reduced in tetraplegic patients to about two-thirds of its normal values. ${ }^{5}$ The above mentioned reduction of chest wall compliance in patients apply to the entire chest wall, but it seems reasonable to speculate that the changes are primarily due to altered stiffness of the rib cage. The chronically reduced physical activity of such patients diminishes the need for large respiratory excursions and is likely to lead to stiffening of tissues and ankylosis in the thoracovertebral and costosternal joints. $^{6,7}$ Regular physical activity could favorably influence the aforementioned stiffness of the rib cage. However, the positive effect of respiratory training is most probably a combination of all the above mentioned mechanisms. According to our results it seems that inspiratory muscle training is a little more effective than expiratory training, which concurs with results obtained by other authors ${ }^{8-10}$ and suggest also the importance of the type of respiratory muscle training. Positive results of respiratory muscle training were obtained in both lying and sitting positions. And it is the latter position that is the most important for the rehabilitation of tetraplegic patients.

From the results in Figures 1 to 4 we can see that there was little increment of measured RT even after the 1 month period in which the patients were without training. This increment may be attributed to the random selection of the order of three 1 month periods in each patient. So some of the patients had the aforementioned period at the beginning of the study, some between the periods of respiratory training and some of them at the end, that is after both 1 month periods of training and we can suppose that some effects were retained after the period of rest on behalf of the gain during the period of training. We could also expect some spontaneous improvements of respiratory capacities in our patients since they were included in the study relatively early after the spinal cord lesion (from 3 to 12 months, 7 months on average). But we can conclude that in our results the possible effects of spontaneous improvement of RT are eliminated because of the previous mentioned random selection of the three 1 month periods in each patient.

Four of our 13 patients had high cervical lesions (above C5), two of them complete and two incomplete. Those with complete lesions were placed on ventilator at the time of injury. However, when we began this study they were no longer using ventilators as the chest wall spasticity which replaces flaccidity thus improving pulmonary function as the more rigid chest wall resists collapse. ${ }^{1}$ These patients' respiratory capabilities remain nevertheless quite poor, FVC was less than $30 \%$ of the expected value, absolute values a little over 1 litre which is similar to observations presented by Mansel and Norman. ${ }^{11}$ The remaining nine patients had a lower cervical cord injury and their respiratory capacities were about double that of the other four patients, confirming the assertion of Kelly and Luce ${ }^{1}$ that the higher the level of the spinal cord lesion, the more profound the respiratory failure. The respiratory capacities of the latter patients are in accordance with that measured by Ledsome and Sharp ${ }^{12}$ who reported an increase in the vital capacity of a group of tetraplegics from 1.5 litres at the time of hospital admission to 2.7 litres 18 weeks later.

Greater RT values were obtained from measurements in the lying position than in the sitting position. This is primarily due to the influence of gravitation on 
the abdominal muscles which stabilise the abdominal content and establish the initial position of the diaphragm during inspiration. The weakness of these muscles is more apparent in an upright (sitting) position. Changes in the chest wall configuration that occur in a seated tetraplegic subject breathing at rest also contribute to reduced RT values in that position; during inspiration, while the abdomen expands, the lower rib cage expands in phase with the abdomen, but there is a decrease in the anteroposterior diameter of the upper rib cage. ${ }^{13,14}$ The flaccid rib cage thus adds to the work of breathing through its paradoxical retraction during inspiration. ${ }^{5}$

One of the greatest problems for tetraplegic patients is ineffective cough which leads to deficient clearance of secretions. This contributes to the high incidence of bronchopulmonary infections and other lung complications. Although the intrinsic properties of the airways are normal in patients with neuromuscular disorders, airway function is modified by increase in lung recoil pressure and reductions in the maximum driving pressures that can be applied at the pleural surface. ${ }^{15,16}$ The effectiveness of cough is severely reduced because of the absence of high flow velocities which depend on the dynamic compression of central airways produced by large positive pleural pressures. Thus, the principal effect of expiratory muscle weakness is reduced cough-induced dynamic compression and therewith the linear velocity of airflow through the large intrathoracic airways is also reduced. ${ }^{5}$

When the four abdominal muscles (rectus abdominis, external oblique, internal oblique and transversus abdominis) contract, they pull the abdominal wall inward and produce an increase in intraabdominal pressure. Because abdominal contents are virtually incompressible, this causes the diaphragm to move cranially into the thoracic cavity. This displacement results, in turn, in an increase in pleural pressure and a decrease in lung volume. The abdominals are thus powerful muscles for expiration and play an important role in activities such as forced expiration and coughing. ${ }^{17}$ If there is a profound weakness in these muscles they cannot contract forcefully. It is well known that with externally applied manual pressure to the abdomen it is possible to help the muscles provide more effective coughing. Our study results confirm this. RT values measured for patients' voluntary effort combined with the therapist's manual pressure during the early phase of expirium were greater than those obtained for patients' unassisted efforts. We thus assumed that we can help weakened abdominal muscles provide a more efficient cough in yet another way, namely by ES. It has been demonstrated that isolated stimulation of the rectus abdominis in healthy humans produces a marked caudal displacement of the sternum and a large decrease in the rib cage anteroposterior diameter. ${ }^{18,19}$ Our results confirm such action in tetraplegic patients. RT measurements were greater when the patient's voluntary effort was combined with ES of abdominal muscles than when it was not. Similar reports were published by Linder. ${ }^{20}$ In our study ES was triggered by the therapist and by the patients themselves. To enable triggering by the patients themselves we employed a wide switch control which was positioned so that patients could press it with their arm extended. Unfortunately some technical difficulties which arose when patients were using this device made it impossible to determine correctly the stimulation onset in regard to the airflow. We believe that precise timing of stimulation is of importance and we intend to investigate this in future work. ES triggered by patients themselves has a great advantage over the manually assisted method yet correct timing for stimulation onset has to be determined and ensured by the device mentioned here; the patient is thereby able to cough when the need to do so arises and not when the therapist happens to be available.

\section{Conclusion}

According to the results of our study we may conclude that respiratory muscle training influences the respiratory capabilities of tetraplegic patients favourably and that ES of the abdominal muscles has potentials to enhance their coughing. It remains open to determine an effective and optimal training of the respiratory muscles and the precise timing for effective triggering of ES by the patients themselves.

\section{References}

1 Kelly BJ, Luce JM. The diagnosis and management of neuromuscular diseases causing respiratory failure. Chest 1991; 99: $1485-1494$

2 Schmidt-Nowara WW, Altman AR. Atelectasis and neuromuscular respiratory failure. Chest $1984 ; \mathbf{8 5}$ : $792-795$.

3 Braun NMT, Arora NS, Rochester DF. Respiratory muscle and pulmonary function in polymyositis and other proximal myopathies. Thorax 1983; 38: 616

4 Braun SR, Giovannoni R, O'Connor M. Improving the cough in patients with spinal cord injury. Am J Phys Med 1984; 63: 1-10.

5 DeTroyer A, Pride NB. The respiratory system in neuromuscular disorders. In: Roussos C, Macklem PT (ed.) The Thorax. Marcel Dekker: New York - Basel. 1985: pp. 1089-1121.

6 Affeldt JE, Whittenberger JL, Mead J, Ferris B Jr. Pulmonary function in convalescent poliomyelitic patients. II. The pressurevolume relations of the thorax and lungs of chronic respiratory patients. $N$ Engl J Med 1952; 247: $43-47$

7 Ferris BG, Mead J, Whittenberger JL, Saxton GA. Pulmonary function in convalescent poliomyelitic patients. III. Compliance of the lungs and the thorax. N Engl J Med 1952; 247: 390-393.

8 Fugl-Meyer AR. A model for treatment of impaired ventilatory function in tetraplegic patients. Scand J Rehab Med 1971; 3: $168-177$.

9 Gross D et al. The effect of training on strength and endurance of the diaphragm in quadriplegia. Am J Med 1980; 68: 27-35.

10 Biering-Sorensen $\mathrm{F}$ et al. Effect of respiratory training with a mouth-nose-mask in tetraplegics. Paraplegia 1991; 29: $113-119$.

11 Mansel JK, Norman JR. Respiratory complications and management of spinal cord injuries. Chest 1990; 97: 1446- 1452.

12 Ledsome JR, Sharp JM. Pulmonary function in acute cervical cord injury. Am Rev Respir Dis 1985; 124: $41-53$.

13 Estenne M, DeTroyer A. Relationship between respiratory muscle electromyogram and rib cage motion in tetraplegia. $\mathrm{Am}$ Rev Respir Dis 1985; 132: 53. 
14 Martola JP, Sant'Ambrogio G. Motion of the rib cage and the abdomen in tetraplegic patients. Clin Sci 1978; 54: 25-32.

15 Danon J, Druz WS, Goldberg NB, Sharp JT. Function of the isolated paced diaphragm and the cervical accessory muscles in C1 quadriplegics. Am Rev Respir Dis 1979; 119: 909-919.

16 Black LF, Hyatt RE. Maximal static respiratory pressures in generalized neuromuscular disease. Am Rev Respir Dis 1971; 103: $641-650$

17 DeTroyer A, Deisser P. The effects of intermittent positive pressure breathing on patients with respiratory muscle weakness. Am Rev Respir Dis 1981; 124: 132 - 137.
18 DeTroyer A, Estenne M. Functional anatomy of the respiratory muscles. Clinics in Chest Medicine 1988; 9: 175-193.

19 Mier A, Brophy C, Estenne M et al. Action of abdominal muscles on rib cage in humans. J Appl Physiol 1985; 58: 1438.

20 Linder SH. Functional ES to enhance cough in quadriplegia. Chest 1993; 103: $166-169$. 